\title{
Valoración de los diferentes tratamientos empleados en la leucoplasia oral
}

\author{
Esparza Gómez GC*
}

\section{RESUMEN}

El tratamiento de la leucoplasia oral (LO) se basa en la premisa de la detección precoz y el manejo activo de estos pacientes para prevenir el desarrollo del carcinoma oral de células escamosas (COCE). No se han definido todavía para esta entidad clínica protocolos de tratamiento estandarizados para reducir el riesgo de transformación maligna. Se han empleado numerosos tratamientos, tanto médicos como quirúrgicos No se ha encontrado ningún tratamiento que sea efectivo para prevenir la transformación maligna; y si bien algunos tratamientos (vitamina A, los retinoides, el betacaroteno y el licopeno) son efectivos para la resolución clínica, ninguno puede prevenir las recidivas y/o la malignización. Tampoco se han comprobado evidencias de que existan ventajas obvias en realizar tratamiento quirúrgico.

Se realiza una revisión de los tratamientos empleados en los últimos años en las LO así como se comentan algunas revisiones realizadas sobre este aspecto..

Palabras clave: Leucoplasia oral, Tratamiento.

\section{SUMMARY}

Treatment of oral leukoplakia (OL) is based on early detection and the active management of these patients in order to prevent the development of oral squamous cell carcinoma (OSCC). Nowadays, does not exist standarized protocols to decrease the risk of malignat transformation. A great number of different medical and surgical treatments had been employed, but none of them has proved to be effective in the prevention of malignant transformation, and although some of them (vitamin A, retinoids, betacarotens and licopene) are effective for the clinical resolution, none of them can prevent relapses and/or malignization. As well, surgical treatment has no showed evidences of advantage.

It had been performed a literature review about the differents treatments employed in recent years in OL and comments about some reviews in this topic are made.

Key words: Oral leukoplakia, Treatment.

Fecha de recepción: Enero 2008.

Aceptado para publicación: Enero 2008.

* Prof. Titular. Dpto de Medicina y Cirugía Bucofacial. Facultad de Odontología. Universidad Complutense de Madrid.

Esparza Gómez GC. Valoración de los diferentes tratamientos empleados en la leucoplasia oral. Av. Odontoestomatol 2008; 24 (1): 105-109.

\section{INTRODUCCIÓN}

El tratamiento de la leucoplasia oral (LO) se basa en la premisa de la detección precoz y el manejo activo de estos pacientes para prevenir el desarrollo del COCE. Dado que la gran mayoría de las LO son asintomáticas, no son mórbidas o letales en si mismas, que el riesgo de transformación maligna es 
relativamente bajo y que muchos sujetos que reciben tratamiento tienen lesiones que nunca evolucionarán hacia cáncer, los tratamientos deberían tener efectos adversos mínimos en cuanto a incidencia y gravedad. El objetivo primario debe ser prevenir la transformación maligna y secundariamente lograr la resolución clínica y la modificación histológica (1).

No se han definido todavía para esta entidad clínica protocolos de actuación estandarizados para reducir el riesgo de transformación maligna. Los más empleados han sido la vigilancia y seguimiento, la modificación de los hábitos de fumar y beber, la quimioprevención con retinoides y carotenos, la extirpación con bisturí frío, láser, criocirugía, terapia fotodinámica y la aplicación tópica de bleomicina y 5-fluoracilo. Tampoco se conoce el valor añadido de la cirugía en la prevención de la malignización en aquellas LO asociadas al tabaco en las que, además de eliminar el hábito, son extirpadas (1).

Los estudios que investigan marcadores biológicos y características histológicas no han encontrado ningún método fiable para identificar que lesión experimentará transformación maligna y cual no (2). Los marcadores clínicos, histológicos y moleculares pueden contribuir a evaluar el riesgo de un paciente para contraer el cáncer; sin embargo no se dispone actualmente de un solo factor predictivo, clínicamente útil y basado en la evidencia, de transformación maligna para las leucoplasias (3-5).

\section{TRATAMIENTOS RECIENTEMENTE EMPLEADOS EN LA LO}

En los últimos años se han ensayado diferentes tratamientos médicos para la LO, entre los que se incluyen: curcumina, (6); galato-epigalocatequina-3 (ECGC) y té verde (7), inhibidor de Bowman-Birk, (8), inhibidores de la COX 2 y EGFR, licopeno $(9,10)$ y fenretidina (11).

Los retinoides se han venido utilizando desde hace bastantes años. Un estudio reciente de Scardina y cols. tras 10 años de experiencia en el tratamiento de las LO con isotretinoino mostró un $85 \%$ reducción de las lesiones y la desaparición de los fenómenos displásicos en ausencia de reacciones adversas (12).
El licopeno, un betacaroteno que se encuentra fundamentalmente en tomates y sandías, ha obtenido resultados esperanzadores. Utilizado a dosis de $8 \mathrm{mg} /$ día ha obtenido respuesta clínica e histológica en el $80 \%$ de los casos por lo que se sugiere que puede ser efectivo en el manejo y tratamiento de la leucoplasia $(9,10)$.

Por lo que respecta a los tratamientos quirúrgicos en todas sus modalidades, durante bastante tiempo se ha considerado que podían ser la solución definitiva para erradicar las LO. Estudios recientes sobre el empleo de la cirugía mediante láser de $\mathrm{CO}_{2}$ para la extirpación de las lesiones han mostrado una tasa de recurrencias del $29 \%$ y una transformación maligna en el 1-4\% de los casos. La escisión parece funcionar mejor en epitelio no queratinizado, mientras que la vaporización obtiene mejores resultados en queratinizado. Con este método la cicatrización suele ser satisfactoria y sin complicaciones $(13,14)$.

Un seguimiento durante 25 años de 252 LO tratadas mediante láser $\mathrm{CO}_{2}$ obtuvo una tasa de recurrencias del $10 \%$ y un $1 \%$ de transformación maligna (15).

Otro estudio piloto reciente comparando la efectividad de la cirugía de láser $\mathrm{CO}_{2}$ frente al de Er: Yag concluía que ambas modalidades parecen tener limitaciones para conseguir de modo predecible una erradicación de la LO. En el grupo de $\mathrm{CO}_{2}$ recurrencias alcanzaron el $25 \%$ a las $38-42$ semanas (16).

Resulta muy interesante comentar en detalle el estudio retrospectivo (1977-1997) de Holmstrump y cols. (17) donde se valoran 269 lesiones de LO en 236 pacientes (seguimiento 1,5-18,6 años) siendo 188 leucoplasias homogéneas (39 extirpadas), 66 leucoplasias no homogéneas (46 extirpadas), 15 eritroplasias (9 extirpadas) y 175 que no se extirparon. De las extirpadas el $13 \%$ sufrieron transformación maligna, más las no homogéneas, (seguimiento medio 7,5 años); de las no extirpadas $16 \%$ desaparecieron espontáneamente y un $4 \%$ sufrieron transformación maligna, más las no homogéneas (seguimiento medio 6,6 años).

Concluyen que a la vista de sus resultados el tratamiento quirúrgico no previno a todas las lesiones de transformación maligna. Además parece que el ries- 
go de desarrollar cáncer, ni se elimina ni se reduce significativamente por el hecho de la intervención quirúrgica. Es más, las lesiones tratadas quirúrgicamente desarrollaron cáncer $(13 \%)$ en una proporción más alta que las no intervenidas (4\%).

El análisis de regresión logística de sus datos muestra además que la mayoría de los factores que caracterizan las lesiones (localización, bordes, presencia o no de displasia, consumo de tabaco, tratamiento quirúrgico) no tienen significación estadística. Tan sólo el tipo clínico (no homogéneas OR: 7) y el tamaño (> $200 \mathrm{~mm}^{2}$ OR: 5,4) parecen tener significación estadística. Es remarcable que el impacto del tamaño apenas había sido constatado previamente en la literatura. Los hallazgos histológicos de la biopsia inicial (grado de displasia us no displasia) no tuvieron significación estadística con el curso de las lesiones.

Además observaron que las localizaciones consideradas tradicionalmente de alto riesgo (borde lingual, cara ventral de la lengua y suelo de boca) no mostraron riesgo mayor que otras localizaciones.

Entre sus conclusiones señalan que no se encuentra ninguna evidencia que soporte que el tratamiento quirúrgico previene frente a la transformación maligna, aunque tampoco se evidencia lo contrario. A pesar de que el efecto de la intervención quirúrgica no tiene significación estadística, los resultados pudieran sugerir, incluso, un riesgo aumentado de transformación maligna tras la cirugía. En este fenómeno, que ya había sido previamente señalado en la literatura, parecen incidir, por una lado que la cirugía podría actuar como un estímulo que promocionaría la transformación, fenómeno que ya había sido publicado al observarse un aumento de la incidencia de carcinomas tras la escisión de mucosa de hámster tratada con carcinógenos (18). También podía deberse al hecho de que las lesiones tuviesen un origen policlonal como sucede en la cancerización de campo $(19,20)$; o bien a que persistieran células estigmatizadas de cáncer fuera de las áreas extirpadas y de las zonas con cambios clínicos e histológicos visibles $(21,22)$.

Finalmente los autores señalan que las LO homogéneas, extirpadas y no extirpadas mostraron unas ta- sas más altas de persistencia que las no homogéneas, y que el $20 \%$ de las no homogéneas desaparecieron sin intervención quirúrgica. Por lo tanto serían necesarias nuevas modalidades de tratamiento y nuevos métodos para predecir la transformación maligna, además serían necesarios ensayos clínicos randomizados, ya que la limitación de los estudios retrospectivos demuestra que, hoy por hoy, no existen ventajas obvias en realizar tratamiento quirúrgico.

Una revisión de los tratamientos empleados (23) observó que tan sólo existían 19 ensayos clínicos, controlados y randomizados, pero no se pudo incluir ningún ensayo quirúrgico. De los estudiados parece que e la vitamina A y betacarotenos son más efectivos que el placebo o la ausencia de tratamiento en la resolución clínica de las lesiones. Pero cuando se informaron, las recidivas fueron frecuentes en todos los ensayos. Hasta la fecha no existen evidencias de que exista un tratamiento efectivo en prevenir la transformación maligna de la leucoplasia. Algunos tratamientos son efectivos en la resolución de las lesiones, pero las recidivas y los efectos adversos son muy comunes.

Finalmente merece la pena analizar la revisión sistemática realizada por Lodi y cols. (24). Tan solo pudo incluir 9 estudios que cumplieran todos los requisitos exigibles (ninguno de ellos con tratamiento quirúrgico). Del análisis efectuado se desprende que existen algunos indicios de que la vitamina $A$, los retinoides, el betacaroteno y el licopeno pueden resolver las lesiones orales completamente; y de que el ácido retinoico puede prevenir el empeoramiento histológico, pero estas conclusiones se basan en un reducido número de pacientes.

También se desprende que la incidencia de la transformación maligna aumenta con la duración del seguimiento por lo que serían necesarios estudios con grupos grandes de pacientes y seguimiento a largo plazo. Como hecho destacable cabe señalar que casi todos los pacientes incluidos en estos estudios eran mascadores de betel, factor de riesgo muy poco habitual fuera del subcontinente indio.

Es notable la ausencia de estudios que comparen la escisión quirúrgica us ningún tratamiento o placebo 
por lo que no pudieron incluirse en la revisión. Las diferentes técnicas quirúrgicas (bisturí, láser, crioterapia) han mostrado resultados sumamente variables y a veces contradictorios. Aunque la mayoría de los clínicos sigue considerando a la cirugía como la primera elección, realmente no hay datos que avalen este supuesto.

Tampoco se han encontrado ensayos que evalúen intervenciones contra los factores de riesgo (ej: tabaco). No se ha encontrado ningún tratamiento que sea efectivo para prevenir la transformación maligna; y si bien algunos tratamientos son efectivos para la resolución clínica, ninguno puede prevenir las recidivas y/o la malignización.

Hasta que las pruebas definitivas sobre el valor predictivo de los marcadores biológicos específicos estén disponibles, la transformación maligna debe considerarse el mejor resultado para evaluar la eficacia del tratamiento.

\section{BIBLIOGRAFÍA}

1. Bascones Martínez A, Esparza Gómez GC, Cerero Lapiedra R, Campo Trapero J.Leucoplasia oral. En: Bascones A, Seoane JM, Aguado A, Suárez Quintanilla JM. Precáncer oral. Madrid: Avances/ Xunta de Galicia, 2003. 111-29.

2. Warnakulasuriya S. Lack of molecular markers to predict malignant potential of oral precancer. J Pathol 2000;190:407-9.

3. Lee JJ, Hong WK, Hittelman WN, et al. Predicting cancerdevelopment in oral leukoplakia: ten years of translational research. Clin Cancer Res 2000; 6: 1702-10.

4. Zhang L, Rosin MP. Loss of heterozygosity: a potential tool in management of oral premalignant lesions? J Oral Path Med 2001;30: 513-20.

5. Zhang L, Williams M, Poh CF, Laronde D, Epstein JB, Durham S, et al. Toluidine blue staining identifies high-risk primary oral premalignant lesions with poor outcome. Cancer Res 2005; 65:8017-21.

6. Cheng AL, Hsu CH, Lin JK, Hsu MM, Ho YF, Shen TS, Ko JY, Lin JT, Lin BR, Ming-Shiang W, Yu HS, Jee SH, Chen GS, Chen TM, Chen CA, Lai MK, Pu YS, Pan MH, Wang YJ, Tsai CC, Hsieh CY. Phase I clinical trial of curcumin, a chemopreventive agent, in patients with high-risk or pre-malignant lesions. Anticancer Res 2001; 21:2895-900.

7. Khafif A, Schantz SP, al-Rawi M, Edelstein D, Sacks PG. Green tea regulates cell cycle progression in oral leukoplakia. Head Neck 1998; 20: 528-34.

8. Armstrong WB, Kennedy AR, Wan XS, Atiba J, McLaren CE, Meyskens FL Jr. Single-dose administration of Bowman-Bark inhinbitor concentrate in patients with oral leukoplakia. Cancer Epidemiol Biomarkers Prev 2000;9:43-7.

9. Singh M, Krishanappa R, Bagewadi A, Keluskar V.Efficacy of oral lycopene in the treatment of oral leukoplakia. Oral Oncol 2004; 40:591-6.

10. Zakrzewska JM. Oral lycopene-an efficacious treatment for oral leukoplakia? Evid Based Dent. 2005;6:17-8

11. Lippman SM, Lee JJ, Martin JW, El-Naggar AK, Xu X, Shin DM, Thomas M, Mao L, Fritsche HA Jr, Zhou X, Papadimitrakopoulou V, Khuri FR, Tran H, Clayman GL, Hittelman WN, Hong WK, Lotan R. Fenretinide activity in retinoid-resistant oral leukoplakia. Clin Cancer Res. 2006;12: 3109-14.

12 Scardina GA, Carini F, Maresi E, Valenza V, Messina P. Evaluation of the clinical and histological effectiveness of isotretinoin in the therapy of oral leukoplakia: ten years of experience: is management still up to date and effective? Methods Find Exp Clin Pharmacol 2006;28:115-9.

13. Ishii J, Fujita K, Komori T. Laser surgery as a treatment for oral leukoplakia. Oral Onco. 2003; 39:759-69. 
14. Ishii J, Fujita K, Munemoto $\mathrm{S}$, Komori $\mathrm{T}$. Management of oral leukoplakia by laser surgery: relation between recurrence and malignant transformation and clinicopathological features. J Clin Laser Med Surg 2004;22:27-33.

15. van der Hem PS, Nauta JM, van der Wal JE, Roodenburg JL. The results of $\mathrm{CO}_{2}$ laser surgery in patients with oral leukoplakia: a 25 year follow up. Oral Oncol 2005;41:31-7.

16. Schwarz F, Maraki D, Yalcinkaya S, Bieling K, Bocking A, Becker J. Cytologic and DNAcytometric follow-up of oral leukoplakia after CO2-and Er:YAG-laser assisted ablation: a pilot study. Lasers Surg Med 2005;37:29-36.

17. Holmstrup P, Vedtofte P, ReibelJ, Stoltze K. Longterm treatment outcome of oral premalignant lesions. Oral Oncology 2006;42:461-74.

18. Maeda H, Kameyama Y. Effect of excisional wounding on DMBA-induced hamster tongue carcinogenesis. J Oral Pathol 1986;15:21-7.

19. Hays GL, Lippman SM, Flaitz CM, Brown RS, Pang A, Devoll R, et al. Cocarcinogenesis and field cancerization: oral lesions offer first signs. J Am Dent Assoc 1995;126:47-51.
20. Scholes AG, Woolgar JA, Boyle MA, Brown JS, Vaughan ED, Hart CA, et al. Synchronous oral carcinomas: independent or common clonal origin? Cancer Res 1998; 58:2003-6.

21. Tabor MP, Brakenhoff RH, Ruijter-Schippers HJ, Kummer CR, Leemans CR, Braakhuis BJ. Genetically altered fields as origin of locally recurrent head and neck cancer: a retrospective study. Clin Cancer Res 2004;10:3607-13.

22. Braakhuis BJ, Leemans CR, Brakenhoff RH. A genetic progression model of oral cancer: current evidence and clinical implications. J Oral Pathol Med 2004; 33:317-22.

23. Lele S. Although leukoplakia responds to some treatments relapses and adverse effects are common. Evidence-Based Dent 2005;6:15-6.

24. Lodi G, Sardella A, Bez C, Demarosi F, Carrassi A. Interventions for treating oral leukoplakia. Cochrane Database Syst Rev. 2006;18,(4): CD001829.

\section{CORRESPONDENCIA}

Germán C. Esparza Gómez

Correo electrónico: medoral@infomed.es 